\title{
Study of Plasma Copeptin as a Predictor of Diabetic Nephropathy in Type 2 Diabetic Patients
}

H.A.Issa, E.S.Ahmed, W.A.Abd-El-Halim and S.E.Yassen

Clinical and Chemical Pathology Dept., Faculty of Medicine, Benha Univ., Benha, Egypt

E-Mail: waleed.ibrahim@fmed.bu.edu.eg

\begin{abstract}
To determine the association of plasma copeptin, a surrogate of vasopressin, with the incidence of Diabetic Nephropathy in Type 2 diabetes mellitus. Early detection of individuals having the highest risk could help avoid this complication. 60 patients with type 2 diabetes mellitus and 20 apparently healthy subjects serving as control were included. Plasma levels of Copeptin and other biochemical parameters were determined. The correlation between copeptin and estimated glomerular filtration rate (eGFR) was investigated. There was a significant association between elevated copeptin concentrations and eGFR decline $(\mathrm{P}=0.001)$. High plasma levels of copeptin were associated with lower ACR $(\mathrm{P}<$ 0.001). As copeptin is emerging biomarker in diagnosis of many diseases ,our results suggest that copeptin could be a useful prognostic marker for kidney function decline in type 2 diabetic patients and by this eventually help prevent kidney complications.
\end{abstract}

Keywords: Diabetes, Diabetic Nephropathy, Glomerular filtration rate, Copeptin, Chronic kidney disease.

\section{Introduction}

Diabetes mellitus is a chronic metabolic disorder characterised by hyperglycaemia resulting from insulin resistance and insufficiency which may be associated with a wide range of complications if untreated such as nephropathy, retinopathy, neuropathy and also increase the risk of cardiovascular disease [1].

Early identification of nephropathy in diabetic patients is crucial because it creates opportunity for preventing the incidence of Diabetic Nephropathy and/or even slows down the process of end-stage renal disease attributed to diabetes [2].

Type 2 diabetes is associated with severe and increasing micro and macrovascular complications [3]. This includes a higher incidence of cardiovascular diseases and risk of cardiovascular death compared to the background population [4].

One major cause of morbidity and mortality in persons with diabetes is diabetic nephropathy, which is the leading cause of chronic kidney disease(CKD) and end-stage renal disease worldwide[5].

Diabetic nephropathy is classically characterized by a progressive increase of albuminuria followed by a decline of glomerular filtration rate (GFR). Of note, GFR loss has been shown to occur independently of albuminuria or even in the absence of it[6].

Even if several risk factors leading to complications in people with diabetes are known such as high blood pressure and dyslipidemia there is still insufficient knowledge which individuals have highest risk to be affected [7].

Arginine vasopressin (AVP), also called antidiuretic hormone affects glucose metabolism by stimulating gluconeogenesis and glycogenolysis in the liver[8].

AVP is very unstable which makes it unsuited to use routinely as a biomarker .On the other hand, copeptin, the C-terminal fragment of arginine vasopressin prohormone represents the release of AVP. It is stable for days after blood withdrawal, can be readily measured and is considered to be a reliable surrogate marker for AVP [9].
High copeptin concentrations have also been associated with development of the metabolic syndrome and diabetes mellitus [10][11]. Furthermore copeptin seems to be a valuable biomarker in the diagnosis of diabetesinsipidus [12], and a reliable surrogate marker for AVP [13].

An increasing body of experimental, pharmacological and epidemiological data supports a causal role of vasopressin (or antidiuretic hormone) in the development and progression of chronic kidney disease (CKD) [14].

\section{Material and methods}

A case control study was conducted at the clinical pathology and internal medicine departments of Benha University Hospitals from November 2018 to May 2019.

\subsection{Patients and laboratory analyses}

This study was conducted on 60 patients with type 2 diabetes mellitus and 20 apparently healthy subjects serving as control.

All patients signed an informed consent to participate in the study. Inclusion criteria of the patients were patients with T2DM, both genders, with and without CKD. Exclusion criteria were causes of CKD other than T2DM, patients with myocardial infarction ,Diabetes insipidus, and any other medical disorder that can affect the study.

All patients underwent an initial clinical evaluation; gender, age (years),socio-economic and demographic factors, diagnostic of T2DM (onset of detection, years , progression and complication). Body mass index (BMI) was calculated as weight $(\mathrm{kg})$ divided by the square height $(\mathrm{m} 2)$. Systolic and diastolic BP values were measured . The diagnosis of T2DM was made according to the criteria of the American Diabetes Association. The diagnosis of CKD was done according to the criteria of the KDOQI Guidelines.

In addition to full history taking and clinical examination, All patients and controls were subjected to laboratory investigations including Complete blood picture , fasting blood sugar, HbA1c, serum creatinine, serum urea, estimated Glomerular filtration rate (eGFR), 
Urinary albumin $\mathrm{mg} / \mathrm{g}$ creatinine (Albumin creatinine ratio), Quantification of Copeptin in serum.

After overnight fast, a sample of $7 \mathrm{~mL}$ of blood was drawn from all patients; $5 \mathrm{~mL}$ were collected in Vacutainer tubes without anticoagulant and $2 \mathrm{~mL}$ in Vacutainer tubes containing EDTA as anticoagulant. Samples were centrifuged at $1500 \mathrm{rpm}$ for $15 \mathrm{~min}$, serum was separated, and immediately frozen at $-20 \mathrm{C}$ until assayed. urine samples were used for the measurement of microalbuminuria or proteinuria.

The biochemical measurements were performed by enzymatic colorimetric methods in an automated Biosystem A15 auto- analyzer . complete blood count (CBC) using cell counter (Sysmex XS-800i), Sysmex Europe GmbH, Bornbarch 1, 22848 Norderstedt, Germany. Copeptin levels was measured with a commercial enzyme immunoassay This was done using human ELISA kit purchased from Bioneovan company., Ltd, Beijing, China.

Albumin in the urine sample causes agglutination of the latex particles with anti-human albumin.The agglutination of the particles is proportional to the albumin concentration and can be measured by turbidimetry.The test was done using BioSystems reagent kit provided by BioSystems, S.A. Costa Brava 30.08030 Barcelona (SPAIN). The study group included the type 2 diabetes participants $(n=60)$. Copeptin concentrations were measured.

\subsection{Statistical analysis}

The collected data were tabulated and analyzed using SPSS version 16 software (SpssInc, Chicago, ILL Company. Categorical data were presented as number and percentages while quantitative data were expressed as mean \pm standard deviation, median, IQR and range. Chi square test ( X2), or Fisher's exact test (FET) were used to analyze categorical variables. Quantitative data were tested for normality using Shapiro-Wilks test, assuming normality at $\mathrm{P}>0.05$.differences among 2 groups regarding nonparametric variables were analyzed by Mann Whitney $\mathrm{U}$ test. Difference among 3 independent means was analyzed using ANOVA for parametric variables or Kruskal Wallis test (KW) for non-parametric ones. Significant ANOVA and KW tests were followed by post hoc multiple comparisons using Bonferroni tests to detect the significant pairs. Non parametric correlations were assessed by Spearman's correlation coefficient (rho). ROC curve was constructed to detect cutoff value of copeptin with optimum sensitivity and specificity in prediction of nephropathy among diabetic patients. Binary logistic regression analysis was run to assess if copeptin is a predictor of diabetic nephropathy. The accepted level of significance in this work was stated at 0.05 ( $\mathrm{P}<0.05$ was considered significant).

\section{Results}

A total of 80 patients were analyzed. patients were classified as 60 patients with diabetes mellitus type 2 and 20 apparently healthy subjects serving as a control group.
Patients were further subdivided into two groups : (Group I) Diabetic patients with nephropathy and (Group II) Diabetic patients without nephropathy.

Table (1) show copeptin level between study groups that was There is highly significant increase in copeptin level when comparing diabetics with nephropathy with diabetics without nephropathy \& control group $(\mathrm{P}<0.015)$.

There was positive significant correlation between serum copeptin level and fasting blood sugar, HbA1c, serum creatinine and urinary albumin / creatinine ratio(ACR) in diabetic patients with nephropathy. There was negative significant correlation between serum copeptin level and age, BMI and eGFR diabetic patients with nephropathy as shown in table Table (2).

There was a high significant association between elevated copeptin concentrations and a decline of eGFR resulting in development of CKD $(\mathrm{P}=0.0001)$.

\section{Results}

This study showed a significant association between elevated copeptin concentrations in type 2 diabetic patients and decreasing eGFR resulting in CKD .Therefore, it is appropriate to draw the conclusion that copeptin is associated with a decrease in eGFR. The results go along with [15] and [16] that reported that positive associations of copeptin with markers of kidney function or with kidney function decline were observed in populations with CKD or at high risk of CKD, such as people with diabetes . However, only limited prospective data are available on the association of plasma copeptin with the risk of newonset CKD in the general population as decalared by [17] and [18].

High levels of plasma copeptin at baseline were associated with an increased incidence of stage $3 \mathrm{CKD}$, with kidney function decline assessed by the KDIGO criterion "certain drop in eGFR" as reported by [19]. [20] and [16] reported that Plasma copeptin was associated with the risk of severe kidney outcomes, including the doubling of plasma creatinine concentration and/or the incidence of ESRD, in people with type 1 or type 2. [21] found that copeptin was associated with hypertension, DM.

A correlation between plasma copeptin levels and decline of renal function estimated with the eGFR and glucose levels was reported in the patients with T2DM as well as there was an association between plasma copeptin levels with the decline of renal function in patients with T2DM. Patients with T2DM in stage 4 or $5 \mathrm{CKD}$, had the highest concentrations of plasma copeptin [15].

It was reported that the key finding was that elevated baseline copeptin was independently associated with increased risk of CKD development in two separate cohorts from the Swedish general population followed for 9 and 20 years, respectively, But they observed significant association between elevated copeptin and increased risk of specified kidney diseases other than CKD including acute tubuleinterstitial nephritis and hydronephrosis, in a populationbased study $(\mathrm{P}=0.008)$ [22]. 
Table (1) Comparing the studied groups regarding copeptin level.

\begin{tabular}{|c|c|c|c|c|c|c|c|c|c|c|c|}
\hline \multirow[t]{2}{*}{ Variable } & \multicolumn{3}{|c|}{$\begin{array}{c}\text { Diabetics with nephropathy } \\
(n=30)\end{array}$} & \multicolumn{3}{|c|}{$\begin{array}{c}\text { Diabetics without } \\
\text { nephropathy }(n=30)\end{array}$} & \multicolumn{3}{|c|}{$\begin{array}{c}\text { Controls } \\
(\mathbf{n}=20)\end{array}$} & \multirow[t]{2}{*}{ KW test } & \multirow[t]{2}{*}{$\mathbf{P}$} \\
\hline & Mean & \pm SD & Range & Mean & \pm SD & Range & Mean & \pm SD & Range & & \\
\hline $\begin{array}{l}\text { Copeptin } \\
\text { (Pmol/L) }\end{array}$ & $56.6 * t$ & 22.8 & $31.5-120.6$ & 48.8 & 22.4 & $28.6-116.3$ & 40.9 & 7.5 & $28-58.1$ & 8.44 & $0.015(\mathrm{~S})$ \\
\hline
\end{tabular}

KW test $\rightarrow$ Kruskal Wallis test. $* \rightarrow$ Significant in comparison with controls $\$ \rightarrow$ Significant in comparison with group without nephropathy. ${ }^{*}$ There is highly significant increase in copeptin level when comparing diabetics with nephropathy with diabetics without nephropathy \& control group $(\mathrm{P}<0.015)$.

Table (2) Correlation between copeptin level and the studied continuous variables among Diabetic nephropathy group.

\begin{tabular}{lcc}
\hline With & \multicolumn{2}{c}{ Copeptin (Pmol/L) } \\
\cline { 2 - 3 } & \multicolumn{2}{c}{ Diabetic Nephropathy group (N=30) } \\
\cline { 2 - 3 } & Rho & P \\
\hline Age (ys) & -0.368 & $0.046(\mathrm{~S})$ \\
BMI (kg/m2) & -0.525 & $0.003(\mathrm{~S})$ \\
SBP (mmHg) & 0.029 & 0.88 \\
DBP (mmHg) & 0.027 & 0.89 \\
FBS (mg/d) & 0.455 & $0.012(\mathrm{~S})$ \\
HbA1C (\%) & 0.501 & $0.005(\mathrm{~S})$ \\
Hb (gm\%) & 0.077 & 0.68 \\
TLC (x103) & 0.011 & 0.95 \\
PLTs (x103) & -0.016 & 0.93 \\
Urea (mg/dl) & 0.294 & 0.11 \\
Serum creat (mg/dl) & 0.551 & $0.002(\mathrm{~S})$ \\
eGFR (ml/min) & -0.580 & $=0.001(\mathrm{HS})$ \\
ACR (mg/gm creat.) & 0.612 & $<0.001(\mathrm{HS})$ \\
\hline
\end{tabular}

BMI, Body mass index; SBP, Systolic blood pressure; DBP, Diastolic blood pressure; FBS, Fasting blood sugar; (HbA1c), Glycosylated hemoglobin; Hb, Hemoglobin; TLC, Total leucocytic count; PLTs, platlets; eGFR, estimated Glomerular filtration rate; ACR, Albumin creatinine ratio.

\section{Conclusion}

As copeptin is emerging biomarker in diagnosis of many diseases, our results suggest that copeptin could be a useful prognostic marker for kidney function decline in type 2 diabetic patients and by this eventually help prevent kidney complications.

Thus, the study supports the strong and independent associations of copeptin with progression to CKD in type 2 diabetic patients that may offer new tools to identify patients with highest risk of progression.

\section{References}

[1] N. Sarwar, P. Gao, S.R. Seshasai et al., Diabetes mellitus, fasting blood glucose concentration, and risk of vascular disease: A collaborative meta-analysis of 102 prospective studies. The Lancet, Vol. 375 , PP. 2215-2222,2010..

[2] J. Wright , A. Vardhan, the problem of diabetic nephropathy and practical prevention of its progression. Br J Diabetes Vasc Dis, Vol. 8, PP. 272272,2008.

[3] D.W. Lam, D Le Roith, The worldwide diabetes epidemic. Current Opinion in Endocrinology, Diabetes, and Obesity, Vol.19, PP. 93-96,2012.
[4] M. Chiha, M. Njeim, E.G. Chedrawy , Diabetes and coronary heart disease: A risk factor for the global epidemic. International Journal of Hypertension, Vol.6, PP. 22-40,2012.

[5] G. Pugliese, Updating the natural history of diabetic nephropathy. ActaDiabetologica, Vol. 51, PP. 905915,2014.

[6] M. Roett, S. Liegl and Y. Jabbarpour , Diabetic Nephropathy-The Family Physician's Role. Am Fam Physician, Vol. 85, PP. 883-889,2012.

[7] I. Hameed, S.R. Masoodi, P.A, Malik et al., Genetic variations in key inflammatory cytokines exacerbates the risk of diabetic nephropathy by influencing the gene expression. Gene, Vol. 661, p. 51,2018.

[8] S. Enhörning, L. Bankir, N. Bouby et al., Copeptin, a marker of vasopressin, in abdominal obesity, diabetes and microalbuminuria: the prospective Malmö Diet and Cancer Study cardiovascular cohort. Int J Obes, Vol. 37, PP. 598-603,2013.

[9] N.G. Morgenthaler, J. Struck, C. Alonso et al., Assay for the measurement of copeptin, a stable peptide derived from the precursor of vasopressin. Clin chem., Vol. 52, PP. 112-119,2006. 
[10] S. Enhorning, T.J. Wang, P.M. Nilsson et al., Plasma copeptin and the risk of diabetes mellitus. Circulation, Vol. 121, PP. 2102-2108,2010.

[11] S. Enhorning, J. Struck, Wirfalt et al., Plasma copeptin, a unifying factor behind the metabolic syndrome. The Journal of Clinical Endocrinology and Metabolism, Vol., 96, PP. 1065-1072,2011.

[12] W. Fenske and B. Allolio, Clinical review: Current state and future perspectives in the diagnosis of diabetes insipidus: A clinical review. The Journal of Clinical Endocrinology and Metabolism, Vol. 97, PP. 3426-3437.2012.

[13] M.D.L.L. Villela-Torres, A.E. Higareda-Mendoza, A. Gómez-García et al., Copeptin Plasma Levels are Associated with Decline of Renal Function in Patients with Type 2 Diabetes Mellitus.Archives of Medical Research, Vol. 49,2018.

[14] R. El Boustany, I. Tasevska, E. Meijer et al., Plasma copeptin and chronic kidney disease risk in 3 European cohorts from the general population. JCI Insight., Vol. 3 , p. 121479,2018 .

[15] M. Pikkemaat, O. Melander, K. Bengtsson Boström, Association between copeptin and declining glomerular filtration rate in people with newly diagnosed diabetes. The Skaraborg Diabetes Register. J Diabetes Complicat., Vol. 29, PP. 1062-1065,2015.

[16] G. Velho, R. El Boustany, G. Lefèvre et al., Plasma copeptin, kidney outcomes, ischemic heart disease, and all-cause mortality in people with long-standing type 1 Diabetes. Diabetes Care, Vol. 39, PP. 2288-2295,2016.

[17] R. Roussel, N. Matallah, N. Bouby et al., Plasma Copeptin and Decline in Renal Function in a Cohort from the Community: The Prospective D.E.S.I.R. Study. Am J Nephrol., Vol. 42, PP. 107-114,2015.

[18] I. Tasevska, S. Enhorning, A. Christensson et al., Increased levels of copeptin, a surrogate marker of arginine vasopressin, are associated with an increased risk of chronic kidney disease in a general population. Am J Nephrol, Vol. 44, PP. 22-28,2016.

[19] A. Levin, M. Tonelli, J. Bonventre et al., Global kidney health 2017 and beyond: a roadmap for closing gaps in care, research, and policy; Lancet, Vol. 390, PP. 1888-1917,2017.

[20] G. Velho, N. Bouby, S. Hadjadj et al., Plasma copeptin and renal outcomes in patients with type 2 diabetes and albuminuria. Diabetes Care, Vol. 6, PP. 3639-3645,2013.

[21] U. Saleem, M. Khaleghi, N.G. Morgenthaler, et al., Plasma carboxy-terminal provasopressin (copeptin): a novel marker of insulin resistance and metabolic syndrome. J Clin Endocrinol Metab., Vol. 94, PP. 2558-2564,2009.

[22] S. Enhörning, A. Christensson, O. Melander, Plasma copeptin as a predictor of kidney disease. Nephrol Dial Transplant., Vol. 34, PP. 74-82,2019. 